\title{
Zum sprachlichen und kulturellen Potenzial von Comics im Unterricht Deutsch als Fremdsprache in Polen ${ }^{1}$
}

Pomimo iż komiks nie jest nowym medium dydaktycznym, jego potencjał językowy i kulturowy pozostaje dotychczas jedynie $\mathrm{w}$ niewielkim stopniu zbadany. Niniejszy artykuł stanowi próbę uzupełnienia tego deficytu. W celu scharakteryzowania komiksu jako medium punktem wyjścia uczyniono rozważania dotyczące pojęcia komiksu oraz procesu jego percepcji. Na tym tle zanalizowane zostaną możliwości dydaktyczne komiksu w nauczaniu języka niemieckiego jako obcego w Polsce.

Obwohl der Comic kein neues Unterrichtsmedium darstellt, mangelt es an theoretischen Ansätzen, die sein sprachliches und kulturelles Potenzial näher betrachten würden. Der vorliegende Beitrag versteht sich als Versuch, dieses Forschungsdefizit auszugleichen. Um den Comic als Unterrichtsmedium zu charakterisieren, werden zunächst Überlegungen zu Begriff und Rezeptionsprozess angestellt. Vor diesem Hintergrund sollen die didaktischen Möglichkeiten von Comics im fremdsprachlichen Deutschunterricht in Polen hinterfragt werden.

Although the comic is not a new didactic medium, there is still a lack of concepts dealing with its linguistic and cultural potential. This article aims to compensate this shortage. In order to characterize the comic as a didactic medium, the concept of comic and the process of comic reception will be considered first. On this background, the didactic potential of comics in German language teaching as a foreign language in Poland will be analyzed.

Der vorliegende Beitrag entstand im Kontext einer Dissertation zum sprachlichen und kulturellen Potenzial von Comics im Fremdsprachenunterricht, an der die Autorin unter Leitung von Prof. Camilla Badstübner-Kizik am Institut für Angewandte Linguistik der Adam-Mickiewicz-Universität Poznań, Lehrstuhl für Kultur- und Mediendidaktik, arbeitet. 
Magdalena Dudzińska

\section{Comic - zur Begriffsbestimmung}

Die Bezeichnung ,Comic' (oder ,Comics') bezog sich ursprünglich auf die lustigen Bildergeschichten, die am Anfang des 20. Jahrhunderts in der amerikanischen Presse veröffentlicht wurden (engl. ,comics` ist die Abkürzung für ,comic strips‘, was als „komische, drollige Bildstreifen“ übersetzt werden könnte; PERSCHON 2006:71). Eine dynamische Entwicklung des Mediums im Kontext einer langen Tradition des Bilderzählens und eine Vielzahl von möglichen wissenschaftlichen Zugangsweisen bewirken, dass ,Comic' als ein Begriff von sehr unscharfen Grenzen erscheint. Insofern hat GRÜNEWALD (2000:4) Recht, wenn er feststellt: „Comic hat sich als Sammelbegriff durchgesetzt - allerdings unscharf und nicht umfassend definierbar." Die Schwierigkeiten mit der Festlegung einer allgemeingültigen Definition hängen auch mit den Bestrebungen zusammen, Comics den schon existierenden Kategorien, wie Bildender Kunst oder Literatur, zuzuordnen. So wird der Comic als bildkünstlerische oder literarische Ausdrucksform angesehen, während er aufgrund seiner spezifischen Merkmale eher als eigenständiges Medium anerkannt werden könnte (vgl. KNIGGE 1991:89; NÄPEL 1998:6; GRÜNEWALD 2010:28). Die Aufgabe der Fremdsprachendidaktik ist nicht unbedingt die Entwicklung einer neuen Definition oder der Einstieg in den laufenden theoretischen Diskurs zu Comics, sondern vielmehr die Bereitstellung einer Beschreibung, die es erlaubt, den Comic als Unterrichtsmittel zu charakterisieren und ihn von anderen didaktisch relevanten Medien abzugrenzen.

In seinem als Comic konzipierten Werk zur Comictheorie definiert MCCLOUD (2001:17) den Gegenstand seiner Untersuchung als „,[z]u räumlichen Sequenzen angeordnete, bildliche oder andere Zeichen, die Informationen vermitteln und/oder eine ästhetische Wirkung beim Betrachter erzeugen sollen“. Als definitionsrelevantes Kriterium stellt sich der sequentielle Charakter dar. ${ }^{2}$ Dieser lässt die Unterscheidung z.B. von der Karikatur (und ihrem Subgenre, dem ,Cartoon') zu, die vorwiegend als Einzelbild konzipiert wird (vgl. NÄPEL 1998:9; PlATTHAUs 2008:115). Das Prinzip der räumlichen

2 Dieser Gedanke wurde schon früher von EISNER (1985, dt. 1995) aufgegriffen, der Comics als „sequential art“ bezeichnet. McClouds (2001:204) Definition knüpft direkt an dieses Konzept an. Eine ähnliche Comic-Auffassung teilen auch LEFÈVRE / DIERICK (1998:12), indem sie folgende Definition vorschlagen: „The juxtaposition of fixed (mostly drawn) pictures on a support as a communicative act“. Die Frage nach dem narrativen Potenzial des Einzelbildes soll hier nicht aufgegriffen werden (vgl. dazu auch GRÜNEWALD 2000:12). 
Anordnung der Bildsequenz dagegen steht in Opposition zum Animationsfilm, der eine zeitliche Sequenz darstellt (vgl. NÄPEL 1998:9). Dieses Verständnis von Comics erlaubt es, unterschiedlich realisierte visuelle Narrationen unter dem Begriff ,Comic‘ zusammenzufassen.

Einen anderen Weg beschreitet dagegen GRÜNEWALD (1984:21), indem er den Terminus ,Comic‘ zurückweist und für die Bezeichnung „das Prinzip Bildgeschichte“ plädiert. Damit ist „die künstlerische Grundhaltung“ gemeint, „mittels Bildern Geschichten zu erzählen“ (GRÜNEWALD 2010:28). Der Comic mit seinen gattungsspezifischen Gestaltungsmerkmalen sei demzufolge als eine Realisierungsmöglichkeit des Erzählprinzips „Bildgeschichte“ zu betrachten. Dies korrespondiert mit dem von EISNER (1998:12) entwickelten und breiter gefassten Begriff „grafisches Erzählen“, den er wie folgt definiert: „Eine allgemeine Bezeichnung für jede Erzählung, die Bilder verwendet, um eine Vorstellung zu vermitteln.“ McClouds minimalistische Definition, so nützlich sie im Hinblick auf die Komplexität des zu beschreibenden Phänomens erscheint, könnte sich jedoch im didaktischen Kontext gerade wegen ihrer Allgemeingültigkeit als zu wenig präzise erweisen. Eine genauere Beschreibung des Begriffs ,Comic‘ ist demzufolge notwendig.

Als besonders treffend erweist sich in diesem Kontext der Definitionsversuch von Dolle-WeinKaufF (1990:326). Als grundlegende Merkmale des ,Comics' sind hier das Vorhandensein von bildlichen und sprachlichen Elementen und die narrative Bildfolge zu betrachten. Neben diesen Eigenschaften sind es auch die Serialität und feststehende Figuren, die für bestimmte Ausprägungen des Comics in seiner geschichtlichen Entwicklung als definitionsrelevant gelten können (vgl. DOLLE-WEINKAUFF 1990:15). Ausschlaggebend ist jedoch die Integration der Schrift ins Bild, hauptsächlich in Form von Sprechblasen und Textblöcken (vgl. DoLLE-WeINKAUfF 1997:312). Dabei wird die Handlung in unterschiedlichem Grade von beiden Zeichensystemen getragen und die Bedeutung gerade durch das Zusammenspiel von piktoralen und verbalen Komponenten vermittelt. Diese Betrachtungsweise weist eine Ähnlichkeit zu McClouds Auffassung von Comics auf. Während aber dessen Definition den parallelen Gebrauch von Bild und Sprache nur andeutet, wird dieser bei Dolle-Weinkauff zum Konstitutivum. Um die Eigentümlichkeit dieser Comicform zu betonen, hat sich für sie die Bezeichnung ,Sprechblasencomic' durchgesetzt (vgl. SACKMANN 2010:7). Dies setzt den Akzent auf die Form der Textpräsentation in Sprechblasen und somit auf die gleichzeitige Sprach-Bild-Codierung. Der Sprechblasencomic kann somit als „Gattung visuell-verbalen Erzählens“ (DOLLE-WEINKAUFF 1997:312) definiert werden. 
Magdalena Dudzińska

So gesehen gilt ,Comic' als Oberbegriff für Erscheinungsformen wie ,Comicstrips', die auch in Tageszeitungen veröffentlicht werden, oder längere Geschichten in Form von Heften und Alben (z.B. ,Graphic Novel'; vgl. GRÜNEWALD 2000:4). Normative Regeln bezüglich der formalen Gestaltung, die eine einfache Zuordnung zur Gattung Comic ermöglichen würden, konnten bisher nicht formuliert werden. Somit liegt die Entscheidung oft beim Rezipienten (vgl. MounAJEd 2009:13; SACKMANN 2010:6). Über die Spezifik der Comicform entscheidet nach DoLLE-WeINKAUfF (1990:326) u. a. der Einsatz von Darstellungsmitteln, die zwar der Bildenden Kunst und der Literatur entnommen wurden, im Comic aber eine neue Qualität erreichen. Gemeint sind Sprechblase, Onomatopöie und Piktogramm. „Comics“, so DoLLE-WEINKAUFF (1990:326) weiter, ,lassen sich im einzelnen nicht immer völlig eindeutig von verwandten Formen des erzählenden Bildes abgrenzen“. Ebenfalls gibt es keine Regel für die Herstellungstechnik. So können Comics nicht nur gezeichnet, sondern auch gemalt oder fotografiert werden und bleiben somit nicht nur den Printmedien verpflichtet (vgl. LEFÈVRE / DIERICK 1998:12; GRÜNEWALD 2000:4; SACKMANN 2010:6). ${ }^{3}$

\section{Zum Rezeptionsprozess von Comics}

Die Rezeption von Comics erscheint als ein komplexer „Prozess des Wahrnehmens, Verarbeitens und Verstehens“ (GRÜNEWALD 2000:37), in dem dem Leser eine aktive Rolle zukommt. Der Erfolg dieses Prozesses wird durch unterschiedliche Faktoren mitbestimmt. Für eine erfolgreiche Kommunikation mit Comics ist in erster Linie die Vertrautheit mit (kulturellen) Konventionen notwendig (vgl. DOELKER 1998:31; SZYŁAK 2000:13). Im europäischen Kulturraum gilt die Leserichtung von links nach rechts und von oben nach unten. Dem Prinzip zufolge werden die Einzelbilder (Panels) im Comic am häufigsten zu Sequenzen und ganzen Seiten organisiert. Japanische Mangas bleiben dagegen der Leserichtung von rechts nach links verpflichtet, was einen ungeübten Leser, wenigstens bei seiner ersten Begegnung mit dieser Comicform, verunsichern kann. Konventionell ist ebenfalls die Comicsprache $^{4}$, die der Leser entsprechend zu deuten wissen muss, wenn die Absicht

3 Neue Möglichkeiten für die Comic-Produktion und Rezeption schaffen auch moderne digitale Medien, wie Computer und Internet (vgl. McCloud 2001a:142f.).

4 Fuchs / Reitberger (1983:50) nennen es „Emblematik der Comics“. 
des Comiczeichners in dem vorprogrammierten Sinn interpretiert werden soll. Dazu gehören in erster Linie bildnerische Ausdrucksmittel (wie Bildmetapher, Symbole, Bewegungslinien und Gestaltung der Sprech- und Denkblasenumrandungen), die zum Teil ausschließlich im Comic vorkommen oder denen aufgrund der Konventionalisierung erst im Comic eine besondere Bedeutung zukommt. „Entscheidend für die Bildgeschichterezeption ist“, so GRÜNEWALD (2000:41), ,die Einzelpanels nicht additiv zu betrachten, sondern verknüpfend, synthetisierend zu lesen.“ Der Leser wird aufgefordert, Veränderungen, die sich von Bild zu Bild vollziehen, durch genaues und zielgerichtetes Betrachten zu erkennen und auf dieser Grundlage die Handlung zu rekonstruieren (vgl. SZYŁAK 2000:14; GRÜNEWALD 2010:19). Die ,synthetisierende' Leistung des Rezipienten besteht hauptsächlich in seinem Vermögen, das Nicht-Dargestellte, den Leerraum (Hiatus) zwischen den Panels imaginativ zu füllen und kausal-logische Zusammenhänge zwischen den Bildern zu erkennen (vgl. GRÜNEWALD 2000:41f., 2010:18; MCCLOUD 2001:73-75). MCCLOUD (2001) versieht seine comictheoretische Studie mit dem Untertitel „die unsichtbare Kunst“, womit er ausdrücklich auf die tragende Rolle dieses Phänomens (der ,Induktion') bei der Rezeption von Comics hinweist. GUNDERMANN (2007:69) verweist jedoch darauf, dass die Vorstellungskraft des Rezipienten allein für das Füllen der Leerstelle nicht ausreicht: „Dass Leserin und Leser den Hiatus überwinden können, liegt hauptsächlich an einem in den Panels vorhandenen Verweissystem, das Hinweise auf die inhaltliche Verknüpfung gibt.“ Auf der Präsentationsebene liefert der Comic selbst „Indizien“ (BREITHAUPT 2002:37), die durch ihr wiederkehrendes Auftreten die Orientierung des Lesers zu steuern vermögen (vgl. GUNDERMANN 2007:69). Als solche können beispielsweise Comicfiguren identifiziert werden, wie es PANDEL (1994:21) vorschlägt, indem er von einem „pikturalen Verweissystem“ spricht.

GUNDERMANN (2007:70) macht des Weiteren auf das „Prinzip der Synästhesie“ aufmerksam, durch das, „obwohl nur ein Reiz, nämlich ein visueller Reiz geboten wird, mehrere Sinne auf diesen Reiz ansprechen“. Wird ein Geräusch mit Hilfe von Onomatopoetika dargestellt, so wird es für den Leser auf diese Art und Weise ,hörbar‘. Die synästhetische Wirkung des Comics ist durch „kulturelle und individuelle Erfahrung und Imagination“ (GuNDERMANN 2007:70f.) des Rezipienten determiniert. Ein anderer Aspekt ist die Fähigkeit des Comics, den Rezipienten emotional zu beeinflussen (vgl. FUCHS / REITBERGER 1983:47; WEIDENMANN 1991:60; GUNDERMANN 2007:71). Dies kommt durch den Einsatz von Perspektive, Einstellungsgröße, Farbe usw. zustande, deren Auswahl darüber entscheidet, inwiefern der Leser 
in das Geschehen miteinbezogen wird (vgl. EISNER 1995:91; GUNDERMANN 2007:71). Die Rezeption von Comics ist ebenfalls im Hinblick auf ihren intermedialen Charakter zu untersuchen. Dem menschlichen Auge bleibt es vorbehalten, zugleich Bilder zu betrachten und Texte zu lesen. Die Verbindung von Bild und Text, die für die Narrationskonstruktion im Comic prägend ist, lässt den Rezipienten zwischen der Simultanität des Bildes, das punktuell präsentiert wird (TULODZIECKI / HERZIG 2004:37), und der linearen Rezeption des Textes schweben. Die Comiclektüre erfordert Vorwissen, das dem Leser gestattet, das Dargestellte entsprechend zu interpretieren (vgl. GRÜNEWALD 2000:45). Dieses Vorwissen schließt u. a. das Wissen um das Medium selbst mit ein. So behauptet GRÜNEWALD (2010:19):

Meist korrespondiert die Interpretationsanforderung mit dem allgemeinen Wissen, mit Alltags- und vertrauter Medienerfahrung der angesprochenen Zeitgenossen; doch je anspruchsvoller, je weniger ,trivial` eine Bildgeschichte ist, je mehr Innovation und kreatives Spiel eingesetzt wurde, um so mehr korreliert unser Verständnis mit unserem Wissen und unserer Interpretationsfähigkeit.

Die Rezeption von Comics kann auch (kultur)spezifisches Wissen erfordern, je nachdem, welche Inhalte thematisiert und wie sie visuell vermittelt werden. Ein kompetenter Comicleser sollte über sogenannte „piktorale Lesefähigkeit“ (GUNDERMANN 2007:72) verfügen, die nur durch einen möglichst intensiven Umgang mit diesem Medium erworben werden kann (vgl. GRÜNEWALD 1991:108).

\section{Comic als Unterrichtsmedium}

Comics sind schon seit langer Zeit Objekt didaktischen Interesses. Aber obwohl sie schon in den 1970er Jahren als Unterrichtsmedium wahrgenommen wurden, stellen ihre Anwendungsmöglichkeiten in der Fremdsprachendidaktik, darunter in der Didaktik Deutsch als Fremdsprache, immer noch ein wenig untersuchtes Forschungsfeld dar. Zu verfolgen sind vereinzelte Beiträge, in denen das didaktische Potenzial von Comics in Ansätzen aufgegriffen wird (vgl. z. B. HeLLWIG / SiEKMANN 1987).

Um eine angemessene Zugangsweise zum Medium Comic zu sichern, ist in erster Linie ihre Verortung im didaktischen Kontext nötig. HECKE / SURKAMP (2010:10) klassifizieren den Comic als eine der Bildsorten, die im Fremdsprachenunterricht Anwendung findet. So gesehen kann der Comic als Bild bzw. Bildfolge behandelt werden und fällt unter die Kategorie der Abbilder, d.h. in seiner Gestaltung soll „eine direkte Ähnlichkeit mit in der Realität 
vorkommenden Dingen und Lebewesen“ (SCHERLING / SCHUCKALL 1992:27) erzielt werden. Wird der Comic als Bildsorte betrachtet, so erweisen sich die Erkenntnisse der Bilddidaktik für comictheoretische Überlegungen als fruchtbar und bieten sich als Grundlage für didaktisch fundierte Analysen an. Dieser Ansatz wird auch in dem vorliegenden Beitrag weiter verfolgt. Es sollte zugleich im Auge behalten werden, dass diese Betrachtungsweise dem Comic nur teilweise gerecht werden kann. Aufgrund seiner Zeichencodierung ist er zwischen verbalen und bildlichen Medien zu verorten und als „Verbundmedium“ zu klassifizieren (vgl. BADSTÜBNER-KIZIK 2006:50 unter Berufung auf Gienow und Hellwig). Über das besondere Potenzial von Comics für das Erlernen einer Fremdsprache könnte gerade das Zusammenspiel von verbalen und bildlichen Komponenten entscheiden.

\section{Comics in Lehrwerken für Deutsch als Fremdsprache}

Um Konzepte didaktischer Nutzung von Comics im fremdsprachlichen Deutschunterricht zu untersuchen, wird hier von einer breit angelegten Lehrwerkanalyse ausgegangen. Diese bietet die Möglichkeit, didaktische Potenziale von Comics im Unterricht aufzuzeigen. Als Untersuchungsgrundlage wurden 94 Lehrwerke für Deutsch als Fremdsprache für alle Schulniveaus (Grundschule, Mittelschule und Lyzeum) des polnischen Bildungssystems ${ }^{5}$ ausgewählt, die sich auf der Liste des polnischen Bildungsministeriums be-

finden. ${ }^{6}$ Für die Analyse leitend waren folgende Fragen:

1) Welchen Stellenwert besitzen Comics in den Lehrwerken für Deutsch als Fremdsprache?

5 Im polnischen Bildungssystem werden folgende Schulstufen unterschieden: Grundschule (poln. ,szkoła podstawowa', 1.-6. Schuljahr), Mittelschule (poln. ,gimnazjum‘, 7.-9. Schuljahr) und Lyzeum (ein Oberschultyp, poln. ,liceum‘, 10.-12. Schuljahr).

6 Es wurden 5 von 20 zugelassenen Lehrwerkreihen für die Grundschule (insgesamt 15 Lehrwerke, $28 \%$ ), 12 von 16 zugelassenen Lehrwerkreihen für die Mittelschule (insgesamt 40 Lehrwerke, $70 \%$ ) und 15 von 23 zugelassenen Lehrwerkreihen für das Gymnasium (42 Lehrwerke, 53 \%) untersucht. Siehe: http://www.men.gov.pl/index.php?option=com_content\&view=article\&id=2222 \&Itemid=79 (20.5.2013). 
Magdalena Dudzińska

2) Inwiefern handelt es sich um authentische ${ }^{7}$ Comics?

3) In welchen didaktischen Kontexten werden Comics eingesetzt?

Die Ergebnisse der Analyse gestatten Aussagen über aktuelle Tendenzen in der Nutzung von Comics im DaF-Unterricht in Polen.

\subsection{Zum Stellenwert der Comics in den Lehrwerken für Deutsch als Fremdsprache}

Die Frage nach dem Stellenwert von Comics in den untersuchten Lehrwerken für Deutsch als Fremdsprache lässt sich aufgrund der zahlenmäßigen Verhältnisse klären. So kann festgehalten werden, dass Comics am häufigsten in Lehrwerken für die Grundschule eingesetzt werden (95 Beispiele). Je höher die Klassenstufe und je älter die Lernenden, desto weniger Comics finden Eingang in die Lehrwerke. In den Lehrwerken für die Mittelschule sind es 84 Beispiele, während sich auf dem Lyzeumsniveau 27 Comics in den untersuchten Lehrwerken finden lassen. Anhand der Häufigkeit des Einsatzes von Comics in den Lehrwerken für Deutsch als Fremdsprache lässt sich somit eine Korrelation mit dem Alter der Lernenden feststellen. Dabei kann vermutet werden, dass bildliche Darstellungen von den Lehrwerkautoren als besonders ansprechend für die jüngsten Lernenden angesehen werden. Zugleich könnte dem das Vorurteil zugrunde liegen, dass der Comic primär als Kindermedium zu betrachten sei, was im Hinblick auf das aktuelle Angebot auf dem Comicmarkt allerdings nicht bestätigt werden kann.

\subsection{Zur Authentizität}

Der zweite untersuchte Aspekt war die Authentizität der eingesetzten Comics. Hier konnte festgestellt werden, dass mehr authentische Comics in den Lehrwerken eingesetzt werden, je höher die Klassenstufe ist (bei gleichzeitiger Abnahme der eingesetzten Comics in den oberen Klassenstufen). In Lehrwerken für die Grundschule sind es ausschließlich Comics, die speziell für die jeweilige Lehrwerkreihe entworfen wurden. In den Lehrwerken für die Mittelschule werden zu über $20 \%$ authentische Comics eingesetzt, während auf dem Lyzeumsniveau diese Zahl auf etwa 50 \% steigt. In den Lehrwerken für die Mittelschule sind Comics von deutschsprachigen Autoren, wie

Als „authentisch“ werden hier Comics bezeichnet, die in einem außerschulischen Kontext entstanden sind und somit nicht speziell für didaktische Ziele erstellt wurden. 
Tom Körner (Comicserie Touché, z.B. in Genial A1:2002:29), Erich Rauschenbach (Lieschen, z.B. in Sowieso 1:1995:95), Manfred Schmidt (Nick Knatterton, z. B. in Pingpong 3:1998:58), Hansrudi Wäscher (Nick. Blendwerk der Hölle, z.B. in Pingpong 3:1998:40-41), Helen von Allmen ([ohne Titel], z.B. in Genial A1:2002:136) und Peter Hürzeler (Heidi und Peter, z.B. in Sowieso 3:1997:117) zu finden. Ebenfalls werden deutsche Fassungen amerikanischer Comics didaktisiert. Dabei handelt es sich um Titel wie Hägar der Schreckliche von Chris und Dik Browne (in den Lehrwerkreihen Sowieso und Pingpong) $)^{8}$ sowie Tarzan von Edgar Rice Burroughs (z. B. in Pingpong 3:1998:15f.)). Inhaltlich handelt es sich hauptsächlich um lustige Comicstrips (Touché, Lieschen, Nick Knatterton, Heidi und Peter, Hägar der Schreckliche) oder Ausschnitte aus längeren Science-Fiction- (Nick. Blendwerk der Hölle) und Abenteuercomics (Tarzan). Unter den didaktisierten Comics sind deutsche Comicklassiker wie Arbeiten von Hansrudi Wäscher und Manfred Schmidt. Bei Helen von Allmen und Peter Hürzeler handelt es sich um zeitgenössische Künstler aus der Schweiz, die nicht ausschließlich Comics zeichnen, sondern auch Karikaturen oder auch malen. ${ }^{9}$

Die eingesetzten Comics - ob authentische oder didaktisierte - stellen keine Fortsetzungsgeschichten dar. Es handelt sich meist um einzelne Comicstrips oder (Foto-)Geschichten, deren Länge eine Seite kaum überschreitet. In der Regel treten sie unsystematisch auf. Eine Ausnahme ist die didaktische Comicreihe Willi und Milli in der Lehrwerkreihe Deutsch aktuell (2004:13). Es kommt ebenfalls vor, dass Lehrwerkfiguren, die die Lernenden beim Lernprozess begleiten, zu Helden einer Comicgeschichte werden (z. B. Angelika und Alexandra im Lehrwerk Fantastisch! 1 2002:132). Auf dem Niveau Mittelschule ist die amerikanische Comicserie Hägar der Schreckliche am stärksten in DaF-Lehrwerken vertreten. Daraus kann der Schluss gezogen werden, dass Herkunft und kulturelle Situierung offenbar kein tragendes Kriterium für die Auswahl von Comics in Lehrwerken darstellen. Als Auswahlkriterien scheinen eher die Thematik, das angenommene Interesse der Lernenden und der sprachliche Schwierigkeitsgrad herangezogen zu werden.

8 Da in Quellenverzeichnissen nicht immer einzelne Autoren, sondern Verlage ausgewiesen werden, die das Urheberrecht an der jeweiligen Comicserie aktuell besitzen, kann die Bestimmung des Comiczeichners in einigen Fällen (insbesondere, wenn die Serie von mehreren Zeichnern fortgesetzt wurde) schwierig sein.

9 Dies lässt sich anhand des Angebots dieser beiden Künstler auf ihren Internetseiten nachvollziehen. Siehe: http://www.peter-huerzeler.ch/, http://www.caricatura. ch/com.cur.htm (19.11.2012). 
Magdalena Dudzińska

In den Lehrwerken für das polnische Lyzeum lässt sich ein ausgewogenes Verhältnis zwischen authentischen und didaktischen Comics beobachten. Neben Comics deutscher Autoren wie Tom Körner (Touché, z. B. in Tangram 1B:1998:129), Vito von Eichborn ([ohne Titel], z.B. in Tangram 1:1999:55), Erich Rauschenbach ([ohne Titel], z.B. in Tangram 1B:1998:101) oder Detlef Surrey (Emil, z.B. in Tangram 2A:1999:30) finden sich auch amerikanische (Walt Disney Company, [ohne Titel], z. B. in Themen neu 1:1997:106) und französische (Jean Marc Reiser, [ohne Titel], z. B. in Stufen international 2:1999:141) Comics jeweils in deutschsprachiger Fassung.

\subsection{Zu den Verwendungskontexten}

Die Verwendungskontexte, auf die im Folgenden zurückgegriffen wird, wurden im Rahmen der Bilddidaktik erarbeitet und beziehen sich somit primär auf den Einsatz von Bildern, sind aber auf die didaktische Nutzung von Comics durchaus übertragbar (vgl. STURM 1991:8f.; REINFRIED 1998:137-141; BADSTÜBNER-KIZIK 2007:45-66; HALLET 2010:33-41). Aus der Analyse geht hervor, dass Comics am häufigsten in den folgenden didaktischen Kontexten zur Erreichung der jeweils sich anschließenden Lernziele Anwendung finden.

\subsubsection{Semantisierung}

In den untersuchten Lehrwerken kommt den Comics auf allen Klassenstufen eine semantische Funktion zu (vgl. REINFRIED 1998:137-141). Sie dienen hauptsächlich als Mittel zur Veranschaulichung von kommunikativen Situationen, als Grundlage für die Präsentation und Einübung von Dialogen sowie als Mittel zur Einführung neuer und Festigung bekannter Lexik. Auf dem Grundschulniveau werden sie häufig am Anfang der Themeneinheit anstelle von rein verbalen Texten eingesetzt. Dabei handelt es sich in den meisten Fällen um einzelne Comicstrips, die keine Fortsetzungsgeschichte darstellen, in denen aber oft festgelegte Figuren über die gesamte Lehrwerkreihe hinweg zu identifizieren bleiben (z.B. die Lehrwerkfamilie in der Lehrwerkreihe Kängookängoo, 2000-2003). Die Figuren erfüllen damit die Funktion von Leitmotiven. Bei allen Klassenstufen werden sehr häufig Illustrationen mit Sprechblasen eingesetzt, die durch formale Gestaltungsmittel zwar eine Ähnlichkeit mit Comics aufweisen, in denen sich aber keine narrativen Strukturen nachweisen lassen. Es handelt sich dabei um Bilder (darunter Fotos) mit integrierten Sprachanteilen, die ebenfalls hauptsächlich eine semantische Funktion erfüllen. In den Lehrwerken für die Grundschule kommen derartige Illustrationen häufig im Einführungsteil des Kapitels vor (vgl. z. B. Ich und 
$d u$ 1:2005:4f.). In den Lehrwerken für Mittelschule und Lyzeum werden Comics außerdem zur Wortschatzpräsentation und -festigung eingesetzt oder liefern den Kontext für weiterführende Aufgaben. Auch hier treten Comics oft anstelle von rein sprachlichen Texten auf.

\subsubsection{Unterstützung der Rezeption und Produktion von Sprache}

In den Lehrwerken für alle Klassenstufen werden Comics zur Schulung von rezeptiven (Hör- und Leseverstehen) und produktiven (Schreiben und Sprechen) Fertigkeiten eingesetzt (vgl. BADSTÜBNER-KIZIK 2007:45-51). Aus der Analyse geht hervor, dass Comics auf dem Grundschulniveau am häufigsten zur Unterstützung des Hörverstehens eingesetzt werden, an zweiter Stelle stehen Übungen zum Leseverstehen, gefolgt von Schreib- und Sprechübungen. In den Lehrwerken für die Mittelschule werden Comics am häufigsten zur Schulung des Leseverstehens genutzt. An zweiter Stelle steht das Schreiben, gefolgt von Sprechen und Hörverstehen. Im Vergleich mit den Lehrwerken für die Grundschule (Hörverstehen) hat sich für diese Schulstufe der Schwerpunkt auf das Leseverstehen verlagert. Auch zur Entwicklung der Schreibfertigkeit werden Comics in höheren Klassenstufen häufiger genutzt. In den Lehrwerken für das Lyzeum werden Comics am häufigsten zur Schulung der Sprechfertigkeit eingesetzt, an zweiter Stelle steht das Schreiben, gefolgt von Leseverstehen und Hörverstehen. In den Lehrwerken für diese Schulstufe lässt sich demzufolge eine klare Verschiebung hin zur Ausbildung produktiver Fertigkeiten beobachten, während in den niedrigeren Klassenstufen ganz deutlich rezeptive Fertigkeiten in der Arbeit mit Comics dominieren.

Im Folgenden wird ein Überblick über häufig eingesetzte Aufgabenformen gegeben, die sich in den didaktischen Konzepten verfolgen lassen, bei denen Comics in DaF-Lehrwerken für alle Klassenstufen eingesetzt werden. Beim Hörverstehen werden Comics hauptsächlich zur Vorentlastung und Kontrolle der Verstehensleistung eingesetzt. Bei vorentlastenden Aufgaben werden die Lernenden beispielsweise häufig zur Betrachtung des Comics vor und während der auditiven Textpräsentation veranlasst. Bei Kontrollaufgaben erweist sich besonders die Bild-Text-Kombination im Comic als vorteilhaft. So können die Lernenden z.B. auf die jeweils sprechenden Personen im Comic zeigen oder im Comictext das Gehörte markieren. Beim Leseverstehen ist zwischen der vorentlastenden Bildarbeit und der Kontrolle der Verstehensleistung zu unterscheiden. In der Phase ,vor dem Lesen' sollten sich die Lernenden die Comicbilder ansehen bzw. sie beschreiben oder kommentieren (auf dem Grundschulniveau auch in der Muttersprache), was das Verstehen 
der sprachlichen Anteile der Comicgeschichte erleichtern soll. Die Übungen zur Kontrolle des Leseverstehens gelten zum Teil auch für rein verbale Lesetexte (z.B. Fragen beantworten). Es gibt zugleich eine Reihe von Übungen, die als comicspezifisch angesehen werden können (z.B. das nächste Panel zeichnen). Es ist dabei anzumerken, dass sich das Leseverstehen auf den Comic als verbal-ikonographischen Text im erweiterten Sinn bezieht. So können die Bilder das Verstehen von sprachlichen Anteilen zwar erleichtern, aber das Erfassen der Comicgeschichte erfordert von dem Lernenden die Fähigkeit zur zusammenführenden Interpretation von Bild und Text. Das Lesen von Comics hängt mit dem Sehen zusammen (vgl. HECKE / SURKAMP 2010:14f.). Zu finden sind auch Übungen, in denen Comics rein sprachliche Texte begleiten und das Textverstehen kontrollieren helfen (z. B. Sprechblasen nach dem Lesen des Textes sinngemäß ergänzen). Bei den Schreibübungen handelt es sich häufig um das Ergänzen von Sprechblasen, sowie schriftliches Nach- und Weitererzählen. Zu finden sind außerdem kreative Übungen, wie das Entwerfen von eigenen Comics. Die Comicsprache imitiert die gesprochene Sprache, was spezifische Schreibübungen ermöglicht: So besteht eine Aufgabe beispielsweise darin, aus elliptischen Sätzen in Sprechblasen vollständige Sätze zu formulieren. Bei den Sprechübungen lassen sich oft Rollenspiele finden. Dies resultiert daraus, dass Comics für die Darstellung von kommunikativen Situationen gut geeignet sind und sich damit als Grundlage für die Einübung von Dialogen anbieten. $\mathrm{Zu}$ verfolgen sind überdies Übungen zum Nach- und Weitererzählen, die von den Lernenden Erzählkompetenz (vgl. dazu NÜNNING / NÜNNING 2003:7-9) erfordern und zugleich zur Entwicklung dieser Kompetenz beitragen können. Es ist anzumerken, dass ein Comic gleichzeitig zur Unterstützung bzw. Entwicklung von mehreren Fertigkeiten eingesetzt werden kann und damit ein komplexes Sprachtraining ermöglicht. Dies gilt für die meisten Comics in allen Klassenstufen.

\subsubsection{Einsatz im Grammatikunterricht}

Comics werden zur Veranschaulichung von grammatischen Phänomenen eingesetzt und liefern den Kontext für die Einübung von grammatischen Strukturen. Im ersten Fall handelt es sich oft um das Auffinden von grammatischen Phänomenen im Comictext, die häufig graphisch hervorgehoben werden. Damit wird den Lernenden zugleich eine mnemotechnische Hilfe bereitgestellt (vgl. STURM 1991:8). Zu den populären Übungsformen gehören das Vervollständigen von Sprechblasentexten (z.B. mit Possessivpronomen, Artikeln oder Präpositionen) und das Ergänzen von comicbezogenen Lücken- 
texten (z.B. entsprechende Verbformen in der Zusammenfassung der Comicgeschichte einsetzen).

\subsubsection{Kulturvermittlung}

Aus der Untersuchung ergibt sich, dass mit Hilfe von Comics auf allen Klassenstufen nur relativ selten kulturspezifische Inhalte vermittelt werden. ${ }^{10}$ Einzelbeispiele lassen sich in den Lehrwerken für die Grundschule finden. Als Vermittlung von landeskundlichem Wissen mit Hilfe von Comics kann hier etwa die bildliche Darstellung von länderspezifischen Orten und Objekten verstanden werden, die in eine Comicgeschichte integriert werden. So unternehmen die Figuren in einer der Comicgeschichten eine Reise nach Wien, von wo aus sie eine Postkarte nach Hause schicken wollen (vgl. Kängookängoo 2.2:2001:29). Durch die Comicbilder bekommen die Lernenden indirekt Informationen darüber, wie Briefkästen in Österreich üblicherweise aussehen, und können dadurch etwa auch zum Vergleich mit dem eigenen Land angeregt werden. In einem anderen Beispiel werden den Lernenden Informationen bezüglich des deutschen Schulsystems sowie der Rituale, die mit dem ersten Schultag verbunden sind (Schultüte), vermittelt, indem sich zwei Comicfiguren über den Schulanfang unterhalten (vgl. Eins, zwei, drei. Teil 3:2000:13). Die bildliche Darstellung solcher eher komplexen Inhalte stellt hier einen deutlichen Vorteil dar. Sie ermöglicht die implizite Vermittlung von landeskundlichen Informationen in imitierten kommunikativen Situationen. Zu finden sind auch Comics, zu deren Themen Osterbräuche (z.B. Eier suchen in Eins, zwei, drei. Teil 3:2001:53) oder Weihnachten (Eins, zwei, drei. Teil 3:2001:34) gehören. Zugleich ist auffällig, dass hierbei keine authentischen Comics eingesetzt werden, was eine ebenso authentische Begegnung mit der ,fremden Welt' im Deutschunterricht eher ausschließt. Die Gründe hierfür sind wahrscheinlich Schwierigkeiten bei der Beschaffung von Materialien, deren sprachliches Niveau den Fähigkeiten der Lernenden entsprechen würde und die sich in das thematische Konzept des jeweiligen Lehrwerkes gut integrieren ließen.

10 An dieser Stelle ist anzumerken, dass die Arbeit mit authentischen Materialien im landeskundlichen DaF-Unterricht an sich als kulturspezifisch angesehen werden könnte. Der Einsatz von authentischen Comics, die auf dem Mittelschulund Lyzeumsniveau gefunden werden konnten (vgl. 4.2), ist aber nicht damit gleichzusetzen, dass in jedem Comic kulturspezifische Inhalte untergebracht werden. In den folgenden Überlegungen geht es darum, inwiefern die Kultur des Zielsprachelandes in den untersuchten Beispielen, authentisch wie didaktisch, reflektiert wird. 
In Lehrwerken für Mittelschule und Lyzeum werden mit Hilfe von Comics ebenfalls nur selten kulturspezifische Inhalte vermittelt oder interkulturelle Lernprozesse initiiert. Es lassen sich nur Einzelbeispiele finden, die deutlich machen, inwiefern Comics zur Unterstützung der bewussten Wahrnehmung oder zum Kulturvergleich veranlassen könnten. Wenn authentische Comics eingesetzt werden, so bleibt in der Regel der Entstehungskontext ausgeblendet. Dies schließt ebenfalls ein, dass sich die Comicautoren größtenteils nur in den Quellenverzeichnissen finden lassen. Als Beispiel kann an dieser Stelle der Ausschnitt aus dem Comic Nick Knatterton genannt werden (Pingpong 3:1998:58), der auf dem Mittelschulniveau eingesetzt wird. Der Comic wurde von dem bedeutenden deutschen Comiczeichner Manfred Schmidt (19131999) in den 1950er Jahren entworfen und war zunächst als Parodie amerikanischer Comics gedacht, die damals in Deutschland sehr populär wurden (vgl. SCHNEIDER 2005). Der Comic wurde immer wieder aufgelegt (letzte Sammelausgabe 2007 im Lappan-Verlag) und auch als Zeichentrickfilm adaptiert. Einer der beliebten Aussprüche von ,Meisterdetektiv‘ Nick Knatterton, „Kombiniere“, avancierte dabei fast zum geflügelten Wort (vgl. SCHNEIDER 2005). In dem Didaktisierungsvorschlag wird der Comic in erster Linie eingesetzt, um die Fertigkeiten Leseverstehen und Sprechen zu üben, während seine kulturelle Botschaft völlig unreflektiert bleibt. Den Lernenden wird der Autor des Comics nicht bekannt gemacht, geschweige denn der weitere Entstehungskontext. Der Weg zu selbstständigem Weitersuchen und Weiterfragen der Lernenden bleibt damit eigentlich verschlossen.

Kulturelle Inhalte können jedoch auch mit Hilfe von speziell für die Lehrwerke erstellten Comics transportiert werden. So wird die Sage von der Entstehung Europas als Comic adaptiert (Europa war ein Mädchen) und in ein Lehrwerk für das polnische Lyzeum integriert (Partnersprache 1:2002:74f.). Die Art und Weise, wie die Thematik dargestellt wird und weiterführende Aufgaben formuliert werden („Was ist Europa heute für dich? Schreib einige Sätze dazu.“, 2002:75) ist mit dem Beitritt Polens zur Europäischen Union in Verbindung zu bringen (das Lehrwerk erschien 2002). Auf der graphischen Ebene wird in dem Comic gezielt mit Stereotypen gearbeitet. So tragen die Französinnen die Mütze der Marianne oder eine Baskenmütze, während der polnische Junge Teile der traditionellen Krakauer Kleidung anhat. Stereotype Darstellungen sollen den Lernenden helfen, die einzelnen Nationalitäten zu erkennen. Sie werden aber nicht explizit thematisiert, was einerseits zu Fehldeutungen, andererseits gar zur Festigung der Stereotype beitragen könnte. Auch an anderer Stelle wird offenbar angenommen, die bildhaften Comicinhalte seien intuitiv zu verstehen, so im Kontext einer Themeneinheit zu 
„Ausbildung und Berufswahl“ (Partnersprache 3:2004: 21), in der die wechselnden Berufsvorstellungen von Jugendlichen über Jahrhunderte hinweg im Comicstil dargestellt werden. Dies ist als eine Reihe von Szenen konzipiert, in denen ein Junge seinen Vater über seinen Traumberuf aufklärt. So werden in einem Bild ein Junge in Matrosenuniform und ein preußischer General im Jahre 1897 dargestellt:

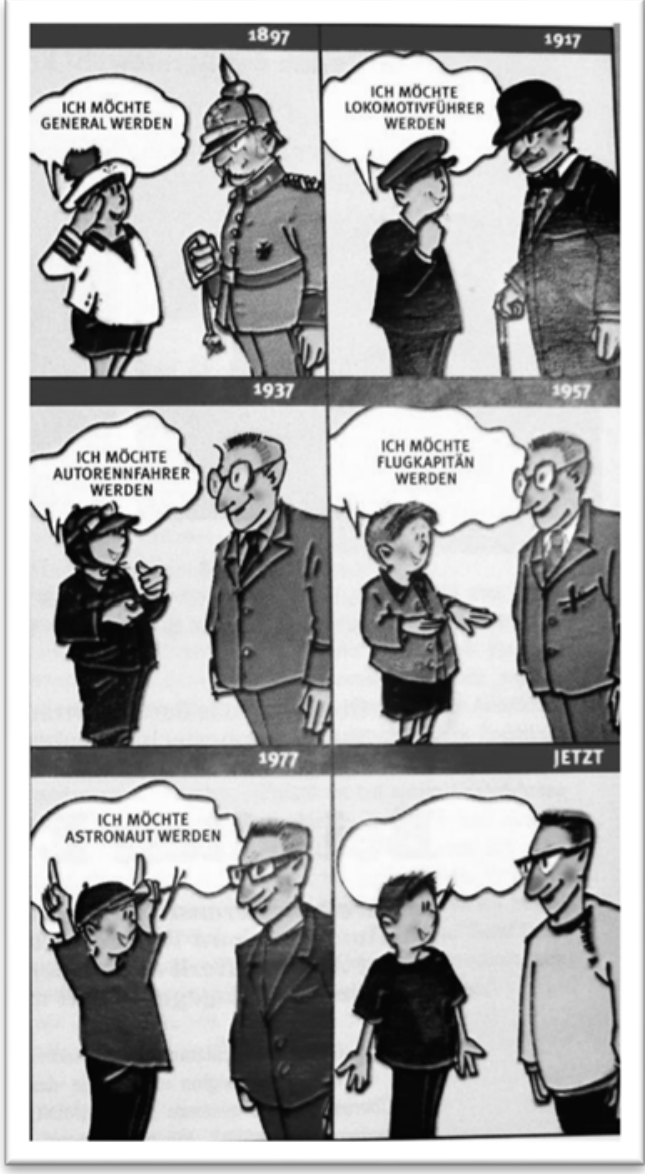

Abb. 1: Aus: Partnersprache 3:2004:21. 
Magdalena Dudzińska

Sicher sind die Lernenden in der Lage, den Mann an seiner Uniform als General zu identifizieren, dennoch dürften Einzelheiten der Uniform wie ,Pickelhaube، oder ,Preußisch Blau' in ihrer historischen Bedingtheit schwer erschließbar bleiben, oder aber - was noch gravierender wäre - unterschwellig vorhandene stereotype Denkmuster verstärken (Pickelhaube - Bismarck Militär - Kulturkampf - Deutsch). Diese Darstellungen lassen auf den ersten Blick auf die Zeitverhältnisse schließen, der kulturelle Kontext aber, und insbesondere der deutsch-polnische Kontext, bleiben völlig unreflektiert. ${ }^{11}$

\section{Comics im Unterricht Deutsch als Fremdsprache - Perspektiven}

Wie oben erläutert, werden Comics am häufigsten in DaF-Lehrwerken auf Grundschulniveau eingesetzt. Dies ergibt sich wahrscheinlich aus der Annahme, dass sich die Leserschaft von Comics hauptsächlich aus Kindern zusammensetzt. Das Angebot an Comics beinhaltet jedoch eine Vielfalt von Genres, die sich an verschiedene Altersgruppen richten und in denen nicht nur triviale Inhalte thematisiert werden. Als prägnantes Beispiel kann an dieser Stelle Maus. A Survivor's Tale (dt. Maus. Die Geschichte eines Überlebenden, 1986) von Art Spiegelman genannt werden, ein Comic, in dem eine persönliche Geschichte über den Holocaust geschildert wird. Maus hat nachhaltig zur Anerkennung des Comics als einer gleichberechtigten Ausdrucksform in den USA beigetragen (1992 wurde der Comic mit dem Pulitzer-Preis ausgezeichnet). Derartige Beispiele lassen sich selbstverständlich auch unter Comics aus dem deutschsprachigen Raum finden. So setzt sich beispielsweise Felix Görmann (Pseudonym Flix) in seinem Comic Da war mal was (2009) mit Erinnerungen an die DDR auseinander und in Lästermaul \& Wohlstandskind. Neue Berliner Geschichten (2011) berichtet Tim Dinter über

11 Vom Standpunkt der fremdsprachigen Kulturdidaktik ist die Zeichnung in dieser Form als fragwürdig einzuschätzen. Die mögliche Intention der Autoren, ein relativ trockenes Thema (Lieblingsberufe) vor veränderlicher ,historischer', offensichtlich deutscher (?) Kulisse attraktiver anzubieten und es dem Schüler zu ermöglichen, sich abschließend selbst in diese Veränderungen einzureihen, muss inhaltlich als kontraproduktiv eingeschätzt werden, vgl. z.B. auch die Darstellung zum Jahr 1937 (Autorennfahrer). Dies soll nicht heißen, dass weder die preußische Teilungsmacht noch der deutsche Faschismus im polnischen DaFUnterricht thematisiert werden sollten (auch in Comicform), allerdings müsste dies mit anderen authentischen Materialien und angemessenem methodischem Instrumentarium erfolgen. 
das Leben von Großstadtmenschen. Im Hinblick auf den Inhalt sind dies Comics, die sich eher an Jugendliche und Erwachsene richten und weniger an Kinder im Grundschulalter. Die behandelten Themen erfordern nicht selten kulturgebundene Kenntnisse, ohne die die Geschichte nur schwer zu erschließen ist. In Bezug auf ihr kulturelles, aber auch sprachliches Potenzial könnte sich der Einsatz von anspruchsvolleren Comics im fremdsprachlichen Unterricht insbesondere bei älteren Jugendlichen als nützlich und sinnvoll erweisen.

Um diese These in der Praxis zu überprüfen, wurde ein didaktischer Vorschlag entwickelt, der an der Oberschule ausprobiert und mit Hilfe einer Umfrage evaluiert wurde. ${ }^{12}$ Die Rückmeldung seitens der Lernenden liefert Informationen bezüglich ihrer bisherigen Comicerfahrungen sowie Erwartungen der Schüler und Schülerinnen gegenüber dem Einsatz von Comics im DaF-Unterricht. Die Umfrage ergab, dass fast die Hälfte der befragten Lernenden häufiger als bisher im Deutschunterricht mit Comics arbeiten möchte. Über 40 \% äußerten sich unentschieden. Dies kann u.a. auf die geringe Erfahrung der Lernenden im Umgang mit diesem Unterrichtsmedium zurückgeführt werden. Über 80 \% der Befragten gab an, im Unterricht Deutsch als Fremdsprache noch nie mit Comics gearbeitet zu haben. Ein einzelner Didaktisierungsvorschlag scheint daher nicht ausreichend zu sein, um eine fundierte Bewertung des Problems zu erreichen. Auf der anderen Seite kann dieser Zustand auch auf die stereotypische Betrachtung von Comics in Polen zurückgeführt werden, wo Comics lange Zeit als minderwertige Literaturform betrachtet wurden. So stellt eine der befragten Personen fest: „Ich bin mir nicht sicher, ob diese Art zu lernen angemessen ist." Lernende, die sich für einen häufigeren Einsatz von Comics im Deutschunterricht aussprechen, sehen Vorteile, die ihnen die Arbeit mit Comics bringen kann. In ihren Begründungen weisen sie z. B. auf folgende Aspekte hin:

- leichtere Wortschatzaneignung (Erhöhung der Gedächtnisleistung): Wir können uns Wörter leichter merken [...].

Mir gefällt die Ausdrucksform. Wir behalten Wörter leicht und merken uns Redewendungen.

- Kontakt mit der , authentischen` deutschen Sprache:

12 An der Untersuchung nahmen 48 Lernende des Lyzeums (zweite Klasse, d.h. 11. Schuljahr) auf dem Niveau A2 teil. Die Untersuchung wurde 2012 an allgemeinbildenden Oberschulen in Leszno und Poznań durchgeführt. 
[...] [D]ank dem Gespräch über Comics lernen wir Redewendungen und den Wortschatz kennen, die in der gesprochenen Sprache, im Alltagsleben nützlich sind.

Comics bieten eine Begegnung mit dem Slang, ohne ,Buchgrammatik' [...].

- $\quad$ Begegnung mit der deutschen Kultur:

Comics bieten eine Begegnung mit [...] der deutschen Kultur. Zusätzlich werden die Unterrichtsstunden interessanter und ,lebhaft‘.

- $\quad$ Abwechslung im Unterricht:

Comics sind eine interessante Alternative im gewöhnlichen Unterricht.

Es ist ziemlich interessant. Wir lernen die Sprache und haben zugleich Spaß.

- Horizonterweiterung,Wissenserwerb:

Ein interessantes Thema. Die Möglichkeit, neue Interessen zu entdecken.

Comics sind eine interessante Art und Weise, wie man neues Wissen erwerben [...] kann.

- $\quad$ Stimulierung der Vorstellungskraft und Förderung der Kreativität:

Comics bieten eine interessante Unterrichtsform. Sie entwickelt die Vorstellungskraft weiter und macht den Unterricht angenehmer.

Comics regen die Phantasie und die Kreativität an.

Die Antworten der Lernenden beziehen sich dabei nicht nur auf sprachgebundene Aspekte. So lässt sich mit Comics mnemonische Hilfe beim Erlernen von unbekanntem Wortschatz leisten, der Zugang zur deutschen Sprache und Kultur erweitern und der Unterricht attraktiver gestalten. Ein Teil der genannten Aspekte weist universellen Charakter auf und gilt somit nicht nur für den DaF-Unterricht, sondern ebenfalls für den Lernprozess im Allgemeinen (z.B. Stimulierung der Vorstellungskraft). Inwiefern Comics den Wissenszuwachs sichern können, hängt dabei von der behandelten Thematik ab. Wenn hier für einen vermehrten Einsatz von Comics im DaF-Unterricht plädiert wird, so werden im Sinne der lernerorientierten Didaktik die Wünsche der Lernenden berücksichtigt. Die Umfrage ergab, dass Lernende am liebsten mit lustigen Comics, Abenteuer-, Fantasy- und Geschichtscomics arbeiten möchten. Als bevorzugte Aufgabenformen wurden mehrheitlich folgende genannt: 
1) neuen Wortschatz mit Comics kennen lernen

2) Sprechblasen ergänzen

3) eine Meinung zu den im Comic thematisierten Sachverhalten äußern

4) eine Comicgeschichte weiterschreiben oder -erzählen

5) Rollenspiele auf der Grundlage von Comics üben und Comicgeschichten inszenieren

6) eigene Comics entwerfen

7) eine Comicgeschichte mündlich oder schriftlich nacherzählen (Angaben nach Häufigkeit der Nennungen).

Dies kann einen Hinweis in Bezug auf die Entwicklung von weiteren didaktischen Vorschlägen zur Nutzung von Comics bei dieser Altersgruppe darstellen.

\section{Schluss}

Die durchgeführte Untersuchung lässt die Behauptung zu, dass Vorschläge zur didaktischen Nutzung von Comics im Unterricht Deutsch als Fremdsprache bisher eher intuitiv entwickelt wurden. Es mangelt an klaren Konzepten, die den Lehrenden bei eigenen Didaktisierungsversuchen behilflich sein könnten bzw. zu diesen anregen würden. Zu hinterfragen wären ebenfalls Kriterien für die Auswahl von Comics, die das Erreichen von kompetenzorientierten Lernzielen des modernen Fremdsprachenunterrichts ermöglichen. Die Offenheit der Lernenden gegenüber dem Comic kann als eine gute und wichtige Voraussetzung für die weitere Erprobung und die Entwicklung von didaktischen Konzepten zum Einsatz von Comics im fremdsprachlichen Deutschunterricht angesehen werden.

\section{Lehrwerke}

Aufderstrasse, Hartmut / Bock, Heiko / Gerdes, Mechthild / Müller, Jutta / MülLER, Helmut (1997): Themen neu 1. Lehrwerk für Deutsch als Fremdsprache. Arbeitsbuch. Ismaning.

Brewińska, Ewa / Joras, Monika / ObidniaK, Dorota / ŚwierczyńsKa, ElżBieta (2002): Partnersprache 1. Podręcznik. [Lehrbuch]. Warszawa.

BREwiŃSKa, Ewa / JoRAs, MONIKA / OBIDNIAK, DOROTA / ŚWIERCZYŃSKA, ELŻBIETA (2004): Partnersprache 3. Podręcznik. [Lehrbuch]. Warszawa.

Dallapiazza, Rosa Maria / V. Jan, EduARD / SchÖNHERR, Til (1998): Tangram. DaF. Kurs- und Arbeitsbuch 1B. Ismaning. 
- (1999): Tangram. DaF. Kurs- und Arbeitsbuch 1A. Ismaning.

Dallapiazza, Rosa Maria / v. Jan, Eduard / BlÜggel, Beate / SchÜmann, AnJa (1999): Tangram. DaF. Kurs- und Arbeitsbuch 2A. Ismaning.

Funk, Hermann / Koenig, Michael / Scherling, Theo / Neuner, Gerd (1995): Sowieso. Deutsch als Fremdsprache für Jugendliche. Kursbuch 1. Berlin/München/ Wien u. a.

Funk, Hermann / Keller, Susy / Koenig, Michael / Mariotta, Maruska / ScherLING, THeo (1997): Sowieso. Deutsch als Fremdsprache für Jugendliche. Arbeitsbuch 3. Berlin/München/Wien u. a.

Funk, Hermann / Koenig, Michael / Koithan, Ute / Scherling, Theo (2002): Genial. Język niemiecki dla młodzieży. Podręcznik A1. [Genial. Deutsche Sprache für Jugendliche. Lehrbuch A1]. Berlin/Warszawa.

Halej, Lucyna / Kozubska, Marta / Krawczyk, Ewa (2000): Eins, zwei, drei. Podręcznik do języka niemieckiego dla klasy trzeciej. [Lehrbuch für die deutsche Sprache in Klasse 3]. Warszawa.

- (2001): Eins, zwei, drei. Zeszyt ćwiczeń dla klasy trzeciej. [Arbeitsbuch für die 3. Klasse]. Warszawa.

Karpeta-Peć, Beata / Peć, Janusz / Wolski, PrzemysŁaw (2002): Fantastisch! Język niemiecki dla gimnazjum (klasa pierwsza). [Deutsche Sprache für die Mittelschule (Klasse 1)]. Warszawa.

Kopp, Gabriele / FröHlich, Konstanze (1998): Pingpong 3. Dein Deutschbuch. Ismaning.

KozUbSKa, Marta / KrawczyK, Ewa / Zastąilıo, Lucyna (2005): Ich und $d u$. Podręcznik do języka niemieckiego dla klasy pierwszej. [Lehrbuch für die deutsche Sprache in Klasse 1]. Warszawa.

Kraft, Wolfgang S. / RybarczyK, Renata / Schmidt, Monika (2004): Deutsch aktuell 1. Kompakt. Język niemiecki dla gimnazjum. [Deutsche Sprache für die Mittelschule]. Poznań.

Stasiak, Halina / AndrzejewsKa, Ewa / Fuks, ANGEliKa (2001): Kängookängoo Deutsch. Podręcznik. Semester 2.2. [Lehrbuch 2.2]. Warszawa.

VORDERWÜLBECKE, ANNE / VORDERWÜLBECKE, KLAUS (1999): Stufen international 2. Deutsch als Fremdsprache für Jugendliche und Erwachsene. Lehr- und Arbeitsbuch. Stuttgart. 
Zum sprachlichen und kulturellen Potenzial von Comics im Unterricht

\section{Literatur}

BADSTÜBNER-KIZIK, CAMILla (2006): Fremde Sprachen - fremde Künste? Bild- und Musikunterricht im interkulturellen Fremdsprachenunterricht. Das Fallbeispiel Deutsch als Fremdsprache in Polen. Gdańsk.

- (2007): Bild- und Musikkunst im Fremdsprachenunterricht. Zwischenbilanz und Handreichungen für die Praxis. Frankfurt (M.).

Breithaupt, Fritz (2002): Das Indiz: Lessings und Goethes Laokoon-Texte und Narrativität der Bilder. In: Hein, Michael / Hüners, Michael / Michaelsen, TORSTEN (eds.): Ästhetik des Comic. Berlin, 37-50.

DoelKer, Christian (1998): Bilder lesen. Bildpädagogik und Multimedia. Donauwörth/Leipzig/Dortmund.

Dolle-WeINKAUfF, BERND (1990): Comics. Geschichte einer populären Literaturform in Deutschland seit 1945. Weinheim/Basel.

- (1997): Comic. In: WeIMAR, Klaus (ed.): Reallexikon der deutschen Literaturwissenschaft. Bd. 1. Berlin/New York, 312-315.

EISNER, WiLl (1995): Mit Bildern erzählen. Comics \& Sequential Art. Wimmelbach.

- (1998): Grafisches Erzählen. Graphic Storytelling. Wimmelbach.

Fuchs, Wolfgang J. / Reitberger, Reinhold (1983): Comics-Handbuch. Reinbek bei Hamburg.

GRÜNEWALD, DieTRICH (1984): Wie Kinder Comics lesen. Frankfurt (M.).

- (1991): Vom Umgang mit Comics. Berlin.

- (2000): Comics. Tübingen.

- (2010): Das Prinzip Bildgeschichte. Konstitutiva und Variablen einer Kunstform. In: GRÜNEWALD, DiETRICH (ed.): Struktur und Geschichte der Comics. Beiträge zur Comicforschung. Bochum/Essen, 11-31.

Gundermann, Christine (2007): Jenseits von Asterix. Comics im Geschichtsunterricht. Schwalbach.

HaLlet, Wolfgang (2010): Viewing Cultures. Kulturelles Sehen und Bildverstehen im Fremdsprachenunterricht. In: HECKE / SURKAMP, 26-54.

HECKE, CAROLA / SuRKAMP, CAROLA (2010): Einleitung. Zur Theorie und Geschichte des Bildeinsatzes im Fremdsprachenunterricht. In: HeCKE, CAROLA / SuRKAMP, CAROLA (eds.): Bilder im Fremdsprachenunterricht. Neue Ansätze, Kompetenzen und Methoden. Tübingen, 9-24.

Hellwig, Karlheinz / SieKmann, Manfred (1987): Authentische Bildergeschichten im Englischunterricht. Teil I: Theoretische Grundlegung. In: Die Neueren Sprachen 6:474-499.

KNIGge, ANDREAs (1991): Faszination durch Bilder, Frustration durch Texte? Comics im heutigen Kulturbetrieb. In: FranzMANN, BODO / HERMANN, INGO / KAGELMANN, HANS-JÜRgEN / ZiTZlsPerger, Rolf (eds.): Comics zwischen Lese- und Bildkultur. Comics Anno. Jahrbuch der Forschung zu populär visuellen Medien. Bd. 2. München, 85-90. 
Magdalena Dudzińska

LefÈvre, Pascal / Dierick, Charles (1998): Introduction. In: Lefèvre, Pascal / Dierick, Charles (eds.): Forging a New Medium. The Comic Strip in the Nineteenth Century. Brussels, 12f.

McCloud, SсотT (2001): Comics richtig lesen. Hamburg.

- (2001a): Comics neu erfinden. Hamburg.

Mounajed, René (2009): Geschichte in Sequenzen. Über den Einsatz von Geschichtscomics im Geschichtsunterricht. Frankfurt (M.)/Berlin/Bern u. a.

NÄPEL, Oliver (1998): Auschwitz im Comic - Die Abbildung unvorstellbarer Zeitgeschichte. Münster.

NÜNNING, VeRA / NÜNNING, ANSGAR (2003): Narrative Kompetenz durch neue erzählerische Kurzformen. In: Der fremdsprachliche Unterricht Englisch 1:4-10.

PANDEL, HANS-JÜRGEN (1994): Comicliteratur und Geschichte. Gezeichnete Narrativität, gedeutete Geschichte und die Ästhetik des Geschichtsbewusstseins. In: Geschichte lernen 37:18-26.

Perschon, Erich ( $\left.{ }^{2} 2006\right)$ : Comics. In: Brunner, Horst / Moritz, Rainer (eds.): Literaturwissenschaftliches Lexikon. Berlin, 71f.

Platthaus, Andreas (2008): Die 101 wichtigsten Fragen: Comics und Manga. München.

REINFRIED, MARCUS (21998): Die Funktion von Bildern in Lehr- und Lernmaterialien. In: JunG, UdO H. (ed.): Praktische Handreichung für Fremdsprachenlehrer. Frankfurt (M.)/Berlin/Bern u. a., 137-142.

SACKMANN, ECKART (2010): Comic. Kommentierte Definition. In: SACKMANN, ECKART: Deutsche Comicforschung 6:6-9. Online verfügbar: http://www. comicforschung.de/pdf/dc10_6-9.pdf (01.11.2010).

SCHERling, THEO / SCHUCKALL, HANS-FriEdRICH (1992): Mit Bildern lernen. Handbuch für den Fremdsprachenunterricht. Berlin/München/Wien u. a.

SchneIDER, MAtThias (2005): Manfred Schmidt: http://www.goethe.de/kue/lit/prj/ com/pck/cks/deindex.htm (17.12.2012).

STURM, DiETRICH (1991): Das Bild im Deutschunterricht. In: Fremdsprache Deutsch 5:5-11.

SZYŁAK, JERZY (2000): Komiks. Kraków.

Tulodziecki, Gerhard / Herzig, BARdo (2004): Handbuch Medienpädagogik. Bd. 2. Stuttgart.

Weidenmann, Bernd (1991): Der exaltierte Code der Comics. In: Franzmann, Bodo / Hermann, Ingo / KAgELMANN, HANS-JÜrgen / ZitZlsPerger, RolF (eds.): Comics zwischen Lese- und Bildkultur. Comics Anno. Jahrbuch der Forschung zu populär visuellen Medien. Bd. 2. München, 60-65. 